\title{
Representing others' preferences in mixed motive games: was Schelling right? *
}

\author{
by Giovanna Devetag ${ }^{\dagger}$ and Massimo Warglien ${ }^{\ddagger}$
}

\begin{abstract}
The available experimental evidence suggests that even two-person normal form games with an elementary action space present substantial degrees of cognitive difficulty. We submit that the relational structure of the players' preferences is a source of complexity of a game. We provide a formal classification of order structures in two-person normal form games, based on the two properties of monotonicity and projectivity, and present an experiment on individual ability to construct a representation of bi-ordered sets isomorphic to the preference structure of paradigmatic normal form games. Experimental results support the hypothesis that relational complexity matters. In particular, they support Schelling's intuition that 'mixed motive games' are harder to represent than 'pure motive' ones. In addition, the experiment shows that most subjects tend to perceive and extract monotonic relations from non-projective ones. We show that individuals' short term memory capacity limitations significantly affect their ability to correctly represent bi-orders. Some connections with Rubinstein's analysis of binary relations in natural language are also shortly discussed.
\end{abstract}

\section{JEL codes: C70, C72}

Keywords: pure motive, mixed motive, preferences, bi-orders, language, cognition, projectivity, monotonicity, short term memory, experiments

${ }^{*}$ Revised version: November 2001. We thank Yaakov Kareev, Marco LiCalzi, Reinhardt Selten, Burkhard Schipper, participants in the CEEL experimental economics workshop in Graz and participants in the ESA world meeting in Barcelona for useful comments and suggestions on a previous draft. The usual disclaimer applies.

${ }^{\dagger}$ Department of Management and Computer Science, University of Trento, Via Inama 5 - 38100, Trento, Italy, devetag@cs.unitn.it.

${ }^{\ddagger}$ Corresponding author. Department of Business Economics and Management, Ca’Foscari University of Venezia, Dorsoduro 1075 - 30123 Venezia, Italy, warglien@unive.it. 


\section{Introduction}

Thomas Schelling reports John Strachey, the former British Defense Minister, telling him that although he had known that conflict could coexist with common interest, he had thought that the two were inherently separable, and had never considered them as part of an integrated structure (Schelling 1980, vi). Strachey's words capture very neatly an important idea in Schelling's (1960) book: representing others' strategic motivations may be a source of cognitive difficulty for players when coordination and conflict motives are intertwined in the same game.

For this purpose Schelling introduces a basic and important distinction between "pure motive" and "mixed motive" games. The former are games in which preferences of players are rank-correlated, as in the prototypical examples of pure coordination games (positive correlation) and conflict games (negative correlation). The latter games present a more complex, non correlated structure of preferences, blending coordination opportunities with antagonistic motivations. The point Schelling makes is that while pure motive games are generally easy to grasp, mixed motive games are puzzling and inherently harder to understand. He strikingly remarks that while our vocabulary is rich of words designating common interest or adversarial relationships, there are no words to designate the relation between players in a mixed motive game: we have a rich lexicon for partners or for opponents, but how to designate someone who is a partner and an opponent at the same time?

While Schelling's typology has received little attention in subsequent developments of game theory (an important exception being Harsanyi, 1977), it has sometimes surfaced in attempts to provide game theoretic prescriptive advice to decision makers. For example, Adam Brandenburger and Barry Nalebuff's (1996) bestseller makes a central argument that managers seldom correctly identify the peculiar mix of competition and cooperation hidden in most business interactions (they feel a revealing need to fill the gap in our dictionaries, coining the hybrid word: co-opetition). Anecdotal evidence from the history of decision making also abounds; for example, Robert McNamara's (1999) recent reappraisal of "missed opportunities" during the Vietnam war provides a rich sample of episodes in which decision makers from both conflicting parties essentially failed to recognize the existence of possible cooperation within conflict. 
In this paper, we experimentally address the cognitive issue raised by Schelling's distinction between pure and mixed motive games. In particular, we want to investigate the extent to which decision making in strategic situations of a mixed motive nature may be affected by the difficulty to correctly represent the underlying game structure in terms of the relations of players' preferences over outcomes. Hence, we focus precisely on possible representational failures that could be at the basis of observed strategic behavior. However, rather than exploring it in the context of a decision making experimental task, we take a more radical semantic stance, and look directly at the cognitive difficulties in representing intertwined order relations which are isomorphic to the preference structures of some classical games. Although such an approach obviously misses important behavioral ingredients typical of an interactive situation, we claim that our experimental strategy may help to disentangle representational factors from other cognitive and behavioral components, and may provide a broader perspective on the difficulties of correctly representing strategic settings. We also propose a finer classification of relational structures in mixed-motive games, introducing the property of projectivity. Projectivity and its complement (non-projectivity) well capture, in our view, the degree of entanglement of multiple order relations, as will be better clarified in the next section.

Our results confirm the appropriateness of such finer classification to understand individual failures in representing complex relational structures. They also confirm that Schelling's insight was essentially correct, and that order relations associated to mixed motive games are significantly more difficult to represent than those mirroring pure motive games. In addition, we show that individuals, when confronted with entangled order relations, tend to perceive out of the whole picture only subsets of elements which are simply (i.e. monotonically) related, as is the case with players' preferences in pure motive games. This preliminary result may suggest that individuals, out of a situation involving both conflict and coordination components, tend to perceive and extract only one of the two relations and ignore the other - or, like John Strachey, to separate them and ignore their interrelations.

Finally, we show that failures in representing order structures isomorphic to mixed motive games are correlated with individual short term memory capacity, and thus seem to be rooted 
in a fundamental cognitive bottleneck.

Section 2 of the paper shortly introduces a formal classification of bi-ordered structures, which can be applied to preference relations in two-person games. Section 3 presents an experiment on the representation of such different structures. Section 4 discusses some implications of our results and suggests further experimental developments.

\section{Bi-orders and preference structures}

A game is usually composed of strategies, players (including Nature), and payoffs which determine the players' preferences over the possible game outcomes. Sources of cognitive difficulty for individuals may in principle arise from any of these elements alone, or, possibly, from their combination. The complexity of the strategy space is indeed an important source of constraints to players' full rationality in games (chess being the paradigmatic example: e.g., Simon and Scheffer, 1992), as partisans of bounded rationality have often suggested, and as an abounding experimental evidence by now confirms.

Much less attention, however, has been paid so far to possible cognitive difficulties arising from the structure of preferences implied by a game ${ }^{1}$.

This diffuse neglect notwithstanding, there is increasing evidence that players can experience serious difficulties in reasoning strategically even in games in which the action space is indeed trivial, as in very simple normal form games (e.g., Stahl and Wilson, 1994; Goeree and Holt, 1999; Devetag, Legrenzi and Warglien, 1999; Costa-Gomez, Crawford, and Broseta, 2001). Since in these games strategic complexity cannot arise from the action space, we suggest that one should look at the structure of players' preferences as an important source of difficulty for strategic thinking in such situations. After all, what distinguishes a game situation from an individual decision making task is the need to jointly take into account both one's own and the other players' preferences, and this may indeed be non-trivial even in those cases in which the strategy space is not exceedingly complicated.

\footnotetext{
${ }^{1}$ Ariel Rubinstein has recently shown some constraints on defining preferences in a simple propositional language: see Rubinstein (2000, ch. 4).
} 
In what follows, we restrict our attention to simple, two-person normal form game structures. A peculiar feature of two-person strategic form games is that the outcomes of strategy profiles (i.e., the cells of the bi-matrix) constitute a bi-ordered set, as the preference order of both players is imposed on them. In order to reason strategically on the game, hence, a player must mentally represent two preference orders, her own and the other player's.

In general, bi-orders can have structures of different complexity. A useful typology of bi-orders, which originated in algebraic linguistics (Marcus, 1967; Schreider, 1975; Mel'cuk, 1988) and which is largely used in the theory of parsing, distinguishes levels of intricacy in the interrelation between two orders on the same set using the properties of monotonicity and projectivity.

Before introducing a few formal definitions, an informal presentation of such properties may be useful.

A bi-order is a pair of order relations (say, $\leftarrow$ and $<$ ) on a set S. Let's assume for the sake of simplicity that both relations are linear orders ${ }^{2}$. A bi-order is monotonic if one relation preserves the order of the other (the bi-order is isotonic) or just reverses it (the bi-order is antitonic). Projectivity can be intuitively expressed by saying that if one writes down the sequence of elements of $\mathrm{S}$ according to the $<$ relation, and draws the arrows directly subordinating (i.e., covering) the same elements according to $\leftarrow$, the $\leftarrow$ covering arrows should never cross each other. Finally, a bi-order is non-projective when it is not projective. Non projectivity can be intuitively expressed by saying that there is no way to arrange the sequence of elements of S according to the $<$ relationship, in such a way that the $\leftarrow$ arrows never cross each other.

Fig. 1 shows an example with four elements and two different types of arrows - continuous and dashed - representing the covering relations of $<$ and $\leftarrow$ respectively.

$$
\text { =Fig. } 1 \text { here }=
$$

\footnotetext{
${ }^{2}$ One can generalize definitions to non strict order relations and to the case in which one of the relations is a tree. See for example Schreider (1975).
} 
More formally:

\section{DEFINITION 1: Monotonic projectivity:}

Let $a_{i}, a_{j} \in \mathrm{S}$, and let $\leftarrow$ and $<$ be two linear order relations defined on $\mathrm{S}$; a doubly ordered set $\mathrm{S}$ is called isotonically projective if:

for $\mathrm{i} \neq \mathrm{j} a_{i}<a_{j}$ iff $a_{i} \leftarrow a_{j}$

It is called antitonically projective if:

for $\mathrm{i} \neq \mathrm{j} a_{i}<a_{j}$ iff $a_{j} \leftarrow a_{i}$

It is called monotonically projective if it is isotonically projective or antitonically projective.

\section{DEFINITION 2: Projectivity:}

Let $a_{i}, a_{j}, a_{k} \in \mathrm{S}$, and let $\leftarrow$ and $<$ be two linear order relations defined on $\mathrm{S}$; furthermore, let $\longleftarrow$ be the covering relation of $\leftarrow^{3}$. A doubly ordered set $\mathrm{S}$ is called projective if one and only one of the following conditions holds:

a) (strict projectivity) for $a_{i} \neq a_{j} \neq a_{k}, a_{i} \hookleftarrow a_{j}$ and $\min \left(a_{i}, a_{j}\right)<a_{k}<\max \left(a_{i}, a_{j}\right)$ imply the relation $a_{k} \leftarrow a_{j}$.

b) (quasi-projectivity) for $a_{i} \neq a_{j} \neq a_{k}, a_{i} \longleftarrow a_{j}$ and $\min \left(a_{i}, a_{j}\right)<a_{k}<\max \left(a_{i}, a_{j}\right)$ imply the relation $a_{i} \leftarrow a_{k}$.

\section{DEFINITION 3: Non projectivity:}

A bi-ordered set is called non projective if it is neither monotonically projective neither projective.

Since monotonic projectivity is nested into projectivity, one can naturally hypothesize a hierarchy of cognitive difficulty: monotonic projective structures are easier to represent than projective (but non monotonic) structures, which in turn are easier to represent than non projective ones. Furthermore, since antitonic projective structures require to reverse one order to obtain the other one, it is reasonable to expect that they may be (slightly) more difficult

\footnotetext{
${ }^{3}$ The covering relation for linear orders is usually defined as follows (Davey and Priestley, 1990). Given an ordered set A, a linear order relation $\leftarrow$ and $a_{i}, a_{j}, a_{k} \in A, a_{j}$ covers $a_{i}\left(a_{i} \leftarrow a_{j}\right)$ if $a_{i} \leftarrow a_{j}$ implies that there are no $a_{k}$ such that: $a_{i} \leftarrow a_{k} \leftarrow a_{i}$.
} 
than isotonic projective structures ${ }^{4}$.

Two-person games are bi-ordered structures: we hypothesize that the cognitive difficulty in representing a game should depend, among other things, on the specific structure of preferences. Pure motive games are monotonically projective structures - in which the two preference relations perfectly coincide - thus they are the easiest to represent; mixed motive games can be of two types: projective ones (like for example "chicken games") or non projective ones (like for example PD's). The latter should be harder to represent, and therefore understand, than the former.

\section{Representing bi-orders}

Our central claim is that there are cognitive constraints in jointly representing multiple order relationships. This constraint seems not specific of game playing only, as the example of language suggests. Thus, we expect to find it in more general representation tasks. In order to test our hypothesis, we designed a very simple experiment in which subjects were provided with a set of objects that could be ordered by two types of order relations and had to select a subset of them that satisfies such order relations. In semantic terms, the task consists in representing a state of affairs (a "world") that satisfies a formula built up with two order relations. In particular, we tested the hypothesis that different bi-orders induce different levels of representational difficulty. As the reader will remind, we hypothesize the following order of difficulty: non projective $\triangleright$ projective (but non monotonic) $\triangleright$ antitonically projective $\triangleright$ isotonically projective, with $\triangleright$ indicating the "more difficult than" relation.

The elements our experimental subjects had to deal with were squares which differed along

\footnotetext{
${ }^{4}$ Linguistics provide some support to this claim in the domain of language. Language is a system that has multiple order structures simultaneously acting on it: there is the sequential order of words in a phrase, as well as many other layers of syntactical (and semantic) order. For example, to parse a phrase we must be able to recognize and process altogether such order relations. Empirical analysis (see Marcus (1967, ch. VI) for a review) has shown that near $100 \%$ of natural language sentences are projective (with non-projective sentences usually confined to literary usage).
} 
the two features of SIZE and COLOR (actually, shades of grey): see fig. 2.

$$
=\text { Fig. } 2 \text { here }=
$$

Squares are very familiar objects, and size and color are equally familiar order relations, henceforth we expected that no peculiar difficulties could arise in understanding them. There was a set of 16 squares, and subjects had to select, out of this set, four squares which would satisfy simultaneously two order relations (size and color) given to them. The experiment was computerized, of the "drag and drop" type (Fig. 8 reports a sample of the computer screen). The upper part of the screen reported the 16 squares from which subjects had to select their "building blocks". Four empty cells in the bottom part of the screen were the TARGET to be filled in with squares taken from the upper part so as to satisfy the formula. Instructions (see Appendix) explained to subjects the meaning of order relations and provided examples.

Instructions also stressed the fact that the particular position of the four squares in the TARGET area of the screen did not matter, as long as the four squares satisfied the two order relations given. In order to perform the task, subjects had simply to click with the mouse on one of the squares in the table and "drag" it into one of the cells in the TARGET.

The pairs of relations given to subjects (see below) were order-isomorphic to payoff structures in four well known $2 \times 2$ strategic games (fig. 3).

$$
=\text { Fig. } 3 \text { here }=
$$

The reader can easily check from fig. 3 that the structure of preferences in a pure coordination game with Pareto-ranked equilibria is isotonically projective. A pure antagonism game corresponds to antitonic projectivity. The projective case is drawn using the chicken game as a template, while the non-projective case is modelled after a Prisoner's Dilemma.

Thus, the following four pairs of order relations were given to subjects: 
- isotonic projectivity (monotonic)

$S(W)>S(Z)>S(Y)>S(X)$

$C(W) \rightarrow C(Z) \rightarrow C(Y) \rightarrow C(X)$

- antitonic projectivity (monotonic)

$S(W)>S(Z)>S(Y)>S(X)$

$C(X) \rightarrow C(Y) \rightarrow C(Z) \rightarrow C(W)$

- projectivity (non-monotonic)

$S(X)>S(Y)>S(Z)>S(W)$

$C(Z) \rightarrow C(Y) \rightarrow C(X) \rightarrow C(W)$

- non-projectivity

$S(X)>S(Y)>S(Z)>S(W)$

$C(W) \rightarrow C(Y) \rightarrow C(Z) \rightarrow C(X)$

$\mathrm{S}$ denotes SIZE and $\mathrm{C}$ denotes COLOR. The four squares are labelled X, Y, Z, and W.

We first ran an experimental session at the University of Venice with a pool of 30 subjects who were students enrolled in a Master in Business Administration. They had a monetary incentive to respond correctly, as they were paid a fixed fee for their participation, plus an amount of $\$ 3$ for each correct answer given in the task. The pool was divided into two subgroups in which the order of presentation of the four tasks was varied, in order to control for transfer of experience effects. Table 1 reports the two differing sequences presented.

$=$ Table 1 here $=$

We then replicated the experiment with identical conditions at the Computable and Experimental Economics Lab of the University of Trento, using a pool of 40 undergraduate students recruited by posting ads at the various department buildings. We first report, in two separate tables, the number of correct responses per task for the Venice and Trento pools respectively (see tables 2 and 3), and distinguishing between the two subgroups. 
$=$ Table 2 here $=$

$=$ Table 3 here $=$

Differences in the number of correct answers between the two sub-groups in each of the four tasks were not found to be statistically significant by a Fisher exact test in both the Venice and Trento pool: therefore we can refer to the pooled data in the last column of the two tables. The first thing to notice by looking at aggregate results is that, notwithstanding the relatively better performance of the Trento subjects in each task, the observed frequencies of correct answers in both pools suggest an order of difficulty that exactly mirrors our hypothesis: relations which are monotonically projective are relatively easy to construct, with the isotonic one easier than the antitonic. On the contrary, non-monotonic projective and non-projective bi-orders seem relatively more difficult. No statistical differences were found between the results obtained in the two experiments (Fisher's exact test), therefore from now on we refer to the pooled data (see table 4).

$=$ Table 4 here $=$

Clearly, aggregate analysis alone is not informative in this experiment, as the single observations (performance in each task) are not independent. Hence, we subsequently performed non-parametric tests on the strings of successes (1) and failures (0) in the four tasks to test against the null hypothesis that these were randomly distributed across subjects.

A Cochran test performed on the four related samples allows to reject the null hypothesis that the correct answers in the tasks are equally distributed at the $1 \%$ significance level ${ }^{5}$. We can hence reject the null hypothesis that the four tasks presented an identical level of difficulty for our subjects.

\footnotetext{
${ }^{5}$ Cochran's $\mathrm{Q}=52.796, p<.000$.
} 
23 subjects made no mistakes in any of the four tasks, while 2 subjects made the highest possible number (4) of mistakes. Disregarding these 25 subjects' performances as noninformative, of the remaining 45 subjects, 35 (78\%) behaved in accordance with our conjecture, i.e., they made mistakes in a way that did not violate our hypothesized hierarchy of difficulty. In particular, 17 subjects made a mistake only in the non-projective task, 9 constructed both monotonic bi-orders correctly but made mistakes in the projective and non-projective tasks, and 9 correctly constructed only the isotonic case. Hence, out of the 16 possible combinations (strings) of successes and failures, the three that are compatible with our hypothesis are highly prominent in the data ${ }^{6}$.

We subsequently made pairwise comparisons by applying a McNemar test. All differences between pairs are statistically significant ${ }^{7}$ except the difference between the antitonic and the projective bi-order $(p=.096)$.

Hence, the data seem to confirm our hypothesis in all but the antitonic-projective pair. In this case, in fact, although observed frequencies suggest the validity of our conjecture, further experiments are needed.

Additional insight can be gathered by conducting an analysis of the most common types of errors that subjects made. While mistakes in the "projective" task show a relatively high variance, mistakes in the "non-projective" task show a rather revealing pattern. In fact, of the thirty-nine subjects who did not answer correctly in this task, twenty (51.3\%) constructed an antitonic bi-order, while fifteen (38.5\%) constructed an isotonic bi-order.

Thus, as we hypothesized, individuals, out of a non-projective pair of relations, tend to simplify their representations by perceiving and extracting monotonic bi-orders.

\footnotetext{
${ }^{6}$ These observed frequencies were not found to be different between the Venice and Trento pool by a Fisher's exact test.

${ }^{7}$ Isotonic-antitonic bi-order: $p=.002$; isotonic-non-projective bi-order: $p=.000$; isotonic-projective biorder: $p=.000 ;$ antitonic-non projective bi-order: $p=.022$; non projective-projective bi-order: $p=.003$; McNemar test.
} 


\section{Short term memory capacity and representation}

Why should some bi-order structures be harder to represent than others? Research in the psychology of mental models (Johnson-Laird 1983) has repeatedly - although not conclusively - suggested that short -term memory constraints may hinder the individual ability to edit a complete, accurate mental representation of a given task-environment. Since the pioneering work of George Miller (1956), it is well-known that we can hold only a limited amount of information active in our short-term memory, which is a basic bottleneck in human information processing. Thus, complex structures may overload individual short-term memory capacity, causing incomplete, over-simplified and often mistaken representations of such structures. The load on short-term memory capacity can be reduced by the ability to compress information or decompose it in smaller components.

Clearly, bi-order structures differ in the way information can be compressed or decomposed. For example, isotone bi-orders can be simply processed as a single order; while antitone ones can be easily obtained by reversing a single order. The case for projective and non-projective bi-orders is less trivial. However, projective structures naturally generate a proper decomposition in a tree of constituents. To see this, it suffices to bracket each pair of elements related by the covering relation $\longleftarrow$ of $\leftarrow$. For example, exploiting the usual order of parentheses and starting from the least element of the chain ordered by $\longleftarrow$, one obtains the bracketing shown in fig. 4 in the case of a projective bi-order:

$$
=\text { Fig. } 4 \text { here }=
$$

This bracketing is "proper", meaning that parentheses are nested. It corresponds to the tree order of the bracketed constituents shown in fig. 5:

$$
=\text { Fig. } 5 \text { here }=
$$

Projectivity implies that a proper bracketing always arises. This follows naturally from the property that the $\longleftarrow$ arrows do not intersect in projective bi-orders. 
On the contrary, it is easy to see that non-projective structures fail to generate such decomposition, as it is shown in fig. 6:

$$
\text { = Fig. } 6 \text { here }=
$$

Consequently, there are good reasons to hypothesize that the short term memory load of editing the representation of a projective bi-order is significantly lowered by the possibility of decomposing it into a tree of constituents (which even a simple stack memory device could easily manage: see Hopcroft and Ullman, 1979). Non-projective bi-orders do not present such a decomposability property, and thus force to consider simultaneously all elements and their order relations.

It has been shown that individuals differ in their short-term memory capacity (Miller, 1956; Baddeley, 1990). If short-term memory capacity limitations are a source of difficulty in representing bi-orders, one should expect that the rate of success of individuals in our experiment may be correlated with individual memory capacity.

In order to test this hypothesis, we conducted on 38 subjects (those participating to the second session of our experiment) a standard Wechsler digit span test for short memory capacity (e.g., Walsch and Betz, 1990). The test consists in asking subjects to repeat a series of digits, which are to be read by the experimenter at the rate of one digit per second. The test is conducted sequentially on two independent sets of digit series of increasing length. For each set, the test stops when the participants fails to correctly repeat a given series. The subject's 'score' in each set is given by the length of the last series that was repeated correctly (so, for example, if a subject fails to correctly repeat a series of length 6 , her score will be equal to 5). The subject's final score is then given by the higher score that was achieved in the two independent sets. Although the score needs not directly reflect the number of 'short term memory slots' available to a subject, it is generally assumed that higher scores correspond to a higher short term memory capacity.

Table 5 reports the correlation between subjects' score in the memory test and the number of correct responses in the representation experiment. We computed the two standard 
Spearman rho and Kendall tau rank-correlation tests. Both tests support our hypothesis of a significant correlation between individual short-term memory capacity and performance in the experiment.

$=$ Table 5 here $=$

\section{Discussion and conclusion}

Was Schelling right, then? Our experiment provides indirect support to the view that not all normal form games are the "same", and that structural complexity matters; we suggest that besides the action space, relational structure is a further source of cognitive difficulty, providing a finer classification of two-person games. "Pure motive" games (i.e. monotonic payoff structures) are easier to represent, and even in the presence of "mixed motive" games, such simpler structures act as irresistible templates of interaction. We also suggest that a further classification, involving the property of projectivity, may be useful, although more data gathering is needed to fully support such claim. Of course, a more direct test requires to move into the domain of experimental games. In a companion paper (Warglien, Devetag and Legrenzi, 2000) we provide some evidence that experimental behavior in simple normal form games may reflect the difficulty to reason through a complete representation of the game structure. Furthermore, a recent experiment by Oechssler and Schipper (2000) lends some support to our hypotheses. In this experiment, subjects play repeatedly a $2 \mathrm{X} 2$ normal form game, in which the payoffs of their opponents are not revealed to them. After 15 rounds, they are asked to guess the order structure of their opponent's payoffs. It turns out that the difficulty of correctly guessing the game structure substantially mirrors our classification of relational complexity; furthermore, an analysis of players' mistakes reveals that they often guess a game structure of lesser ordinal complexity than the 'real one', while the opposite never happens. 
In addition, we submit that monotonic structures may indeed be the prevailing templates of bi-orders available in memory. In a classroom experiment, students asked to provide examples of four arbitrary objects satisfying simultaneously two arbitrary order relations of the kind depicted in fig. 1 had no difficulties in finding examples for monotonic bi-orders (such as "richer is happier" or "larger towns are less healthy"); but they found it almost impossible to provide examples for non-projective bi-orders. This point reinforces the result that in our experiment subjects unable to provide a solution to the non-projective case resorted to monotonic orderings of the squares. Returning to games, it also suggests that in incomplete information games "friends" and "enemies" may be the most natural player types.

Further research would need to identify more precisely a causal relation between failures to represent the mixed motive nature of strategic situations when this is present, and certain behavioral patterns. Some encouraging evidence in this sense comes from a recent experiment on complex multi-issue negotiations (Hyder, Prietula, and Weingart, 2000). The authors of the study observe that negotiators rarely achieve a Pareto-optimal solution to a given negotiation problem, and they argue that the reason lies in their incorrect 'default' representation of the situation as a zero-sum game. In fact, representing the game as zero-sum would trigger the almost exclusive use of distributive negotiation tactics (i.e., tactics aimed at achieving unilateral concessions from the other party) at the expense of integrative tactics, which would instead facilitate the achievement of agreements resulting in gains for both parties involved. Hence, the use of specific behavioral strategies conducting to sub-optimal agreements seems to derive, according to the authors, by an original failure of players to represent the mutual gain area in the space of solution points.

In a different although related direction, the evidence of short term memory capacity effects on representation of complex structures may point at an important cognitive source of behavioral heterogeneity. While short term memory capacity limitations have often been invoked in discussions on bounded rationality, there is indeed very little empirical research aimed at testing how such limitations affect economic behavior and, in particular, strategic interaction (a few recent exceptions include Rapoport and Budescu, 1992, and Kareev, 1992). 
Our results further encourage a systematic exploration of such issue in economically relevant settings.

Finally, our results may provide some complementary cognitive ground to Ariel Rubinstein's (1996) argument on the prevalence of linear order structures in discourse. Rubinstein claims that linear orders have some efficiency properties (in indicating an element out of a set, in being informative about a relation on a set, in minimizing the number of examples necessary to describe a relation) that justify the higher frequency with which such structures appear in natural language. Clearly, one can construct a structure-preserving map from a monotonic bi-order to a linear order, either directly (as in the isotonic case) or with an intermediate step by reversing one of the two order relations (as in the antitonic case). The same cognitive constrains that make monotonic bi-orders easier to represent may underlie the prevalence of linear orders in natural language. Projectivity is a more complex case: no simple way to reduce it to a single linear order can be found. Yet, projectivity can be thought of as a kind of compatibility between order relations, simplifying the task of managing bi-orders in short term memory. The relevance of the projectivity property in natural language suggests that further connections with Rubinstein's argument are worth seeking. 


\section{References}

[1] Baddeley, A.D. (1990): Human Memory: Theory and Practice, Hillsdale, NJ: Erlbaum Associates.

[2] Brandenburger, A.M., and J.N. Nalebuff (1996): Co-opetition, New York, NY: Doubleday.

[3] Costa-Gomez, M., V. Crawford, and B. Boseta (2001): "Cognition and Behavior in Normal-form Games: An Experimental Study", Econometrica, 69, 1193-1235.

[4] Davey B.A., and H.A. Priestley (1990): Introduction to lattices and order, Cambridge, UK: Cambridge University Press.

[5] Devetag, M.G., P. Legrenzi, and M. Warglien (1999): "Focusing in naive game playing", in A. Vandierendonck, in Deductive reasoning and strategies, ed. by G. Devooght, G. d'Ydewalle, and I.W. Schaeken, Hillsdale, NJ: Erlbaum Associates.

[6] Goeree, J.K., C.A. Holt (1999): "Ten Little Treasures of Game Theory and Ten Intuitive Contradictions", Working Paper, University of Virginia, Department of Economics. Forthcoming, American Economic Review.

[7] Harsanyi, J.C. (1977): Rational Behavior and Bargaining Equilibrium in Games and Social Situations, Cambridge, UK: Cambridge University Press.

[8] Hopcroft, J.E., and J.D. Ullman (1979): Introduction to automata theory, languages and computation, Reading (Mass): Addison Wesley.

[9] Hyder, E.B., M.J. Prietula, and L.R. Weingart (2000): “Getting to Best: Efficiency versus Optimality in Negotiation", Cognitive Science, 24(2), 169-204.

[10] Johnson-Laird, P.N. (1983): Mental Models, Cambridge, UK: Cambridge University Press.

[11] Kareev, Y. (1992): "Not that bad after all: Generation of random sequences",Journal of Experimental Psychology: Human Perception and Performance, 18, 1189-1194. 
[12] Marcus, S. (1967): Algebraic Linguistics: Analytical Models, NY: Academic Press.

[13] McNamara, R. (1999): Argument without an end, Public Affairs.

[14] Mel'cuk, I. A. (1987): Dependency Syntax: Theory and Practice. Albany, NY: State University of New York Press.

[15] Miller, G. (1956): “ The magical number seven, plus or minus two. Some limits of our capacity for processing information", Psychological Review, 63, 81-97

[16] Oechssler, J. and B. Schipper (2000): "Can You Guess the Game You're Playing?", Discussion Paper 11/2000, University of Bonn, Department of Economics.

[17] Rapoport, A. and D.V. Budescu (1992): "Generation of random series by humans: A two-person zerosum game paradigm", Journal of Experimental Psychology: General, 121, $352-363$.

[18] Rubinstein A. (1996): "Why are certain properties of binary relations relatively more common in natural language?", Econometrica, 64, 343-356.

[19] Rubinstein, A. (2000): Economics and Language, Cambridge, UK: Cambridge University Press.

[20] Schelling, T. (1980): The Strategy of Conflict, Second Edition, Cambridge, Mass.: Harvard University Press.

[21] Schreider, J. A. (1975): Equality, resemblance and order, Moscow, USSR: MIR Publishers.

[22] Simon H.A. and J. Schaeffer (1992): "The Game of Chess", in: Handbook of Game Theory with Economic Applications, vol. 1, ed. by R. J. Aumann and S. Hart, The Netherlands: Elsevier Science Publishers, 1-17.

[23] Stahl, D.O., and P.W. Wilson (1994): "Experimental Evidence On Players' Models of Other Players", Journal of Economic Behavior and Organization, 25, 309-327. 
[24] Walsh, W.B., and N.E. Betz (1990): "Tests and Assessment", Englewood Cliffs, NJ: Prentice Hall.

[25] Warglien, M., M.G. Devetag, P. Legrenzi (2000): "Mental models of games: Reasoning through others' preferences", Working Paper CEEL, University of Trento, Department of Economics. 


\section{APPENDIX: INSTRUCTIONS}

In the following experiment you will be asked to answer some questions regarding order relations between elements. An order relation, as the term itself indicates, allows some elements of a set to be ordered according to a certain characteristic.

For example, an order relation can be defined according to SIZE: given a set of rectangles, I can say whether rectangle $\mathrm{X}$ is bigger, smaller or equal to rectangle $\mathrm{Y}$; I can also order a set of rectangles from the smallest to the biggest, and vice-versa.

In the following experiment we will ask you to respond some questions about objects according to which two order relations can be defined: one on the basis of SIZE, the other on the basis of COLOR.

We will use the canonical symbols of order relations:

$$
><=
$$

In the case of SIZE, the meaning of the three symbols is obvious and intuitive. For example, the expression $X>Y$ indicates that element $\mathrm{X}$ is bigger than element $\mathrm{Y}$.

In the case of COLOR, you will be proposed four colors: black, white, and two variations of gray. It will be set by convention that the symbol > means "darker than".

In the following tasks, SIZE will be indicated by the letter S, and COLOR by the letter C. Two objects can be defined according to both characteristics. For example, in the following case

$$
=\text { Fig. } 7 \text { here }=
$$

the left circle $(\mathrm{X})$ is bigger than the right circle $(\mathrm{Y})$, but the right circle is darker than the left circle. This double order relation will be expressed in the following way: $S(X)>S(Y)$, and $C(X)<C(Y)$.

Obviously, saying that $S(X)>S(Y)$ is equivalent to saying that $S(Y)<S(X)$. Therefore, the two notations will be used interchangeably. 
In the experiment you will be presented four different pairs of order relations with regard to SIZE and COLOR, with each pair being defined over four elements (squares). The four squares will always be indicated with the letters $\mathrm{X}, \mathrm{Y}, \mathrm{W}$ and $\mathrm{Z}$, while color and size will be denoted with $\mathrm{C}$ and $\mathrm{S}$.

The task will be computerized. Your computer screen will visualize a set of $\mathbf{1 6}$ squares of different colors and sizes, and four empty cells. You will have to fill in the four empty cells with 4 squares (chosen out of the 16) which, according to you, satisfy the pair of order relations that will be provided to you. In order to accomplish the task, you will simply have to click with your mouse on the chosen squares and drag them to the empty cells in the TARGET. For each correct answer, you will be assigned 50 points, which will be converted in cash at the exchange rate of L. 100 per point and paid to you privately at the end of the experiment.

The four empty cells are numbered from 1 to 4 so that the software can recognize them. However, the specific position of the single squares in the cells is irrelevant. In other words, the four squares that you choose can be placed in the empty cells in any position you prefer. It is only important that they satisfy the pair of order relations assigned.

Further, we ask you to carefully read the single pairs of relations given. In this type of experiments it is easy to commit mistakes my simply misreading the data.

In order to begin the experiment, you will have to insert your identification number in the "number" window on your screen and then click OK. After this, the screen will display a set of squares on the left and some written text on the right. Before the actual experiment starts, you will go through a brief training session.

Please, we ask you to do the experiment in silence. Thank you.

$=$ Fig. 8 here $=$ 


\begin{tabular}{c|c|c}
\hline \hline sequence & antit. proj. & non proj. \\
& isot. proj. & proj. \\
& non proj. & isot. proj. \\
& proj. & antit. proj. \\
\hline \hline
\end{tabular}

Table 1: The two sequences presented

\begin{tabular}{|c|c|c|c|c|c|}
\hline \multirow[b]{2}{*}{ N. of correct answers } & \multicolumn{2}{|c|}{ group $1(\mathrm{~N}=14)$} & \multicolumn{2}{|c|}{ group $2(\mathrm{~N}=16)$} & \multirow{2}{*}{$\frac{\text { Tot. } \mathrm{N}=30}{\text { perc. }}$} \\
\hline & number & perc. & number & perc. & \\
\hline Isot.proj. & 13 & $93 \%$ & 15 & $94 \%$ & $93 \%$ \\
\hline Antit. proj. & 9 & $64 \%$ & 12 & $75 \%$ & $70 \%$ \\
\hline Proj. & 7 & $50 \%$ & 10 & $62.5 \%$ & $56.7 \%$ \\
\hline Non proj. & 6 & $42.8 \%$ & 6 & $37.5 \%$ & $40 \%$ \\
\hline
\end{tabular}

Table 2: Number and percentages of correct answers in the four tasks in the Venice pool

\begin{tabular}{c|c|c|c|c|c}
\hline & \multicolumn{2}{|c|}{ group 1 $(\mathrm{N}=20)$} & \multicolumn{2}{c|}{ group 2 $(\mathrm{N}=20)$} & Tot. N=40 \\
\hline \hline N. of correct answers & number & perc. & number & perc. & perc. \\
\hline Isot.proj. & 19 & $95 \%$ & 19 & $95 \%$ & $95 \%$ \\
Antit. proj. & 15 & $75 \%$ & 18 & $90 \%$ & $82.5 \%$ \\
Proj. & 15 & $75 \%$ & 14 & $70 \%$ & $72.5 \%$ \\
Non proj. & 10 & $50 \%$ & 9 & $45 \%$ & $47.5 \%$ \\
\hline \hline
\end{tabular}

Table 3: Number and percentages of correct answers in the four tasks in the Trento pool 


\begin{tabular}{c|c}
\hline \hline task & correct answers \\
\hline \hline isotonic & $66(94.3 \%)$ \\
antitonic & $54(77 \%)$ \\
projective & $46(65.8 \%)$ \\
non-projective & $31(44.3 \%)$ \\
\hline
\end{tabular}

Table 4: Correct answers pooled across sessions

\begin{tabular}{c|c|c}
\hline \hline \multicolumn{2}{c}{ Correlation coefficient } & Significance (one-tailed) \\
\hline \hline Kendall's tau & .310 & .05 \\
Spearman's rho & .362 & .05 \\
\hline \hline
\end{tabular}

Table 5: Correlation coefficients between individual score in the Wechsler digit span test and the number of correct responses in the experiment
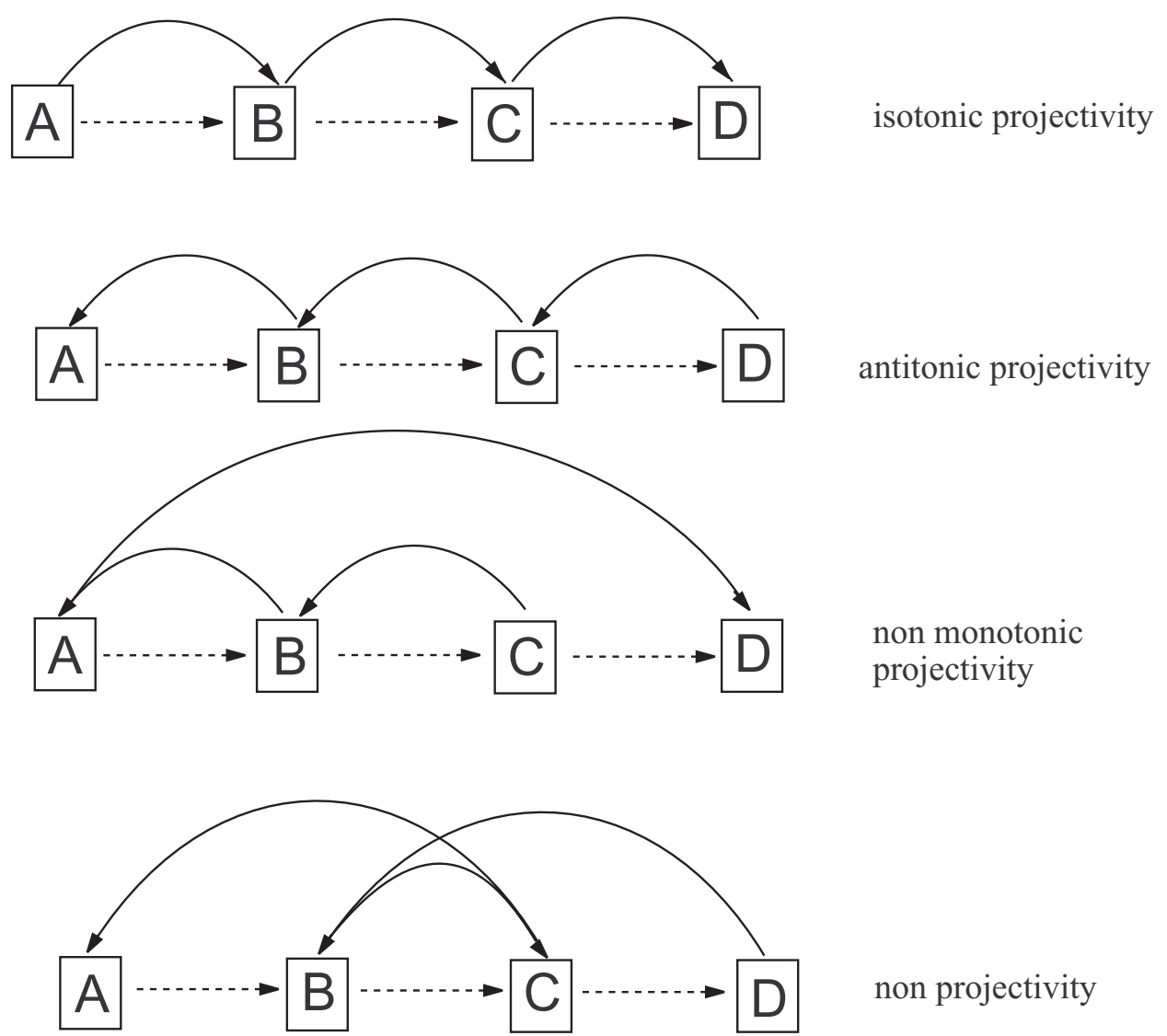

Figure 1: Four examples of bi-order structures 

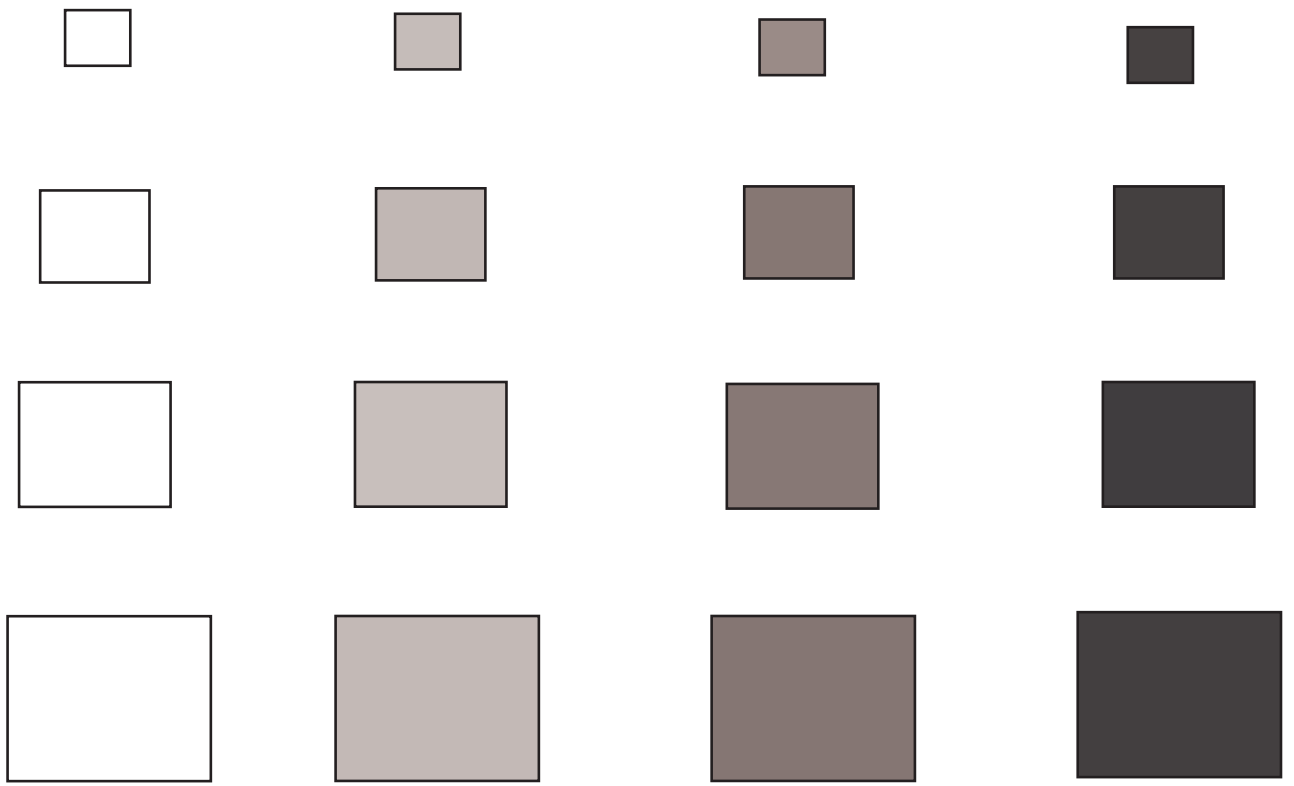

Figure 2: The 16 squares given to subjects

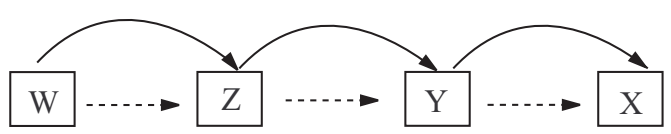

\begin{tabular}{|r|r|}
\hline 4,4 & 2,2 \\
\hline 3,3 & 1,1 \\
\hline
\end{tabular}

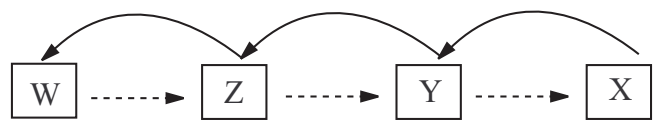

\begin{tabular}{|l|r|}
\hline 4,1 & 2,3 \\
\hline 3,2 & 1,4 \\
\hline
\end{tabular}

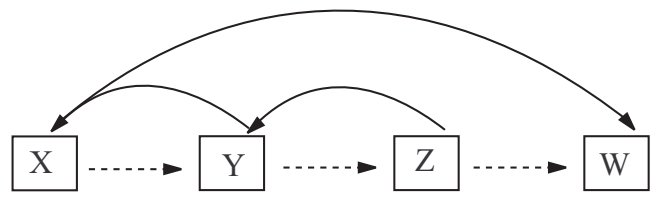

\begin{tabular}{|l|l|}
\hline 3,3 & 2,4 \\
\hline 4,2 & 1,1 \\
\hline
\end{tabular}

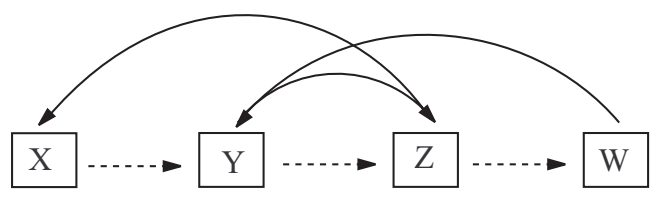

\begin{tabular}{|l|l|}
\hline 3,3 & 1,4 \\
\hline 4,1 & 2,2 \\
\hline
\end{tabular}

Figure 3: The four experimental tasks 


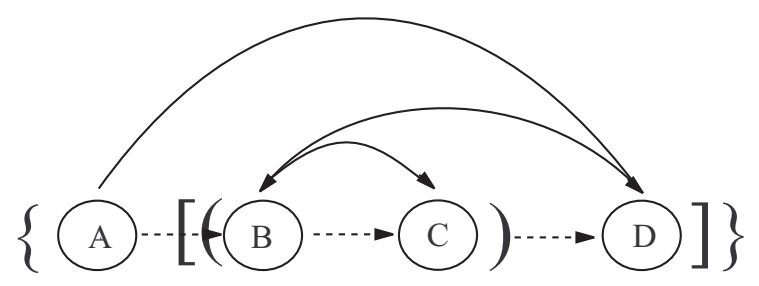

Figure 4:

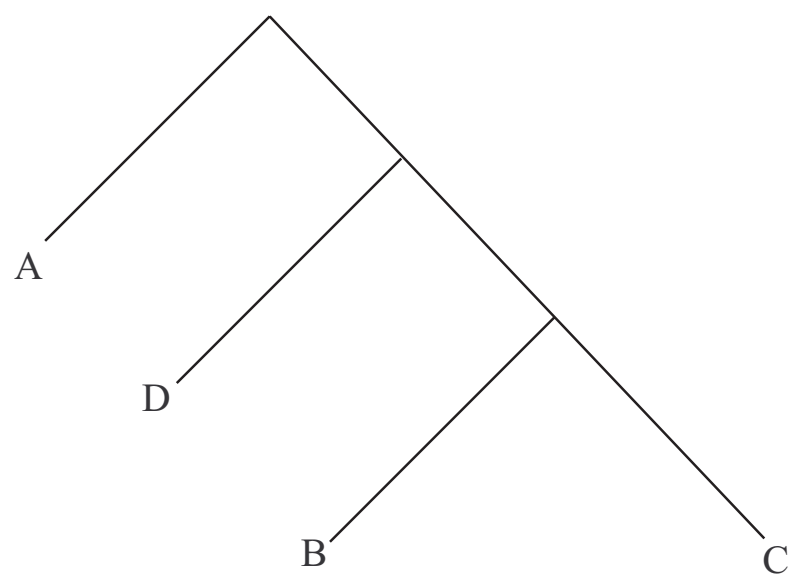

Figure 5:

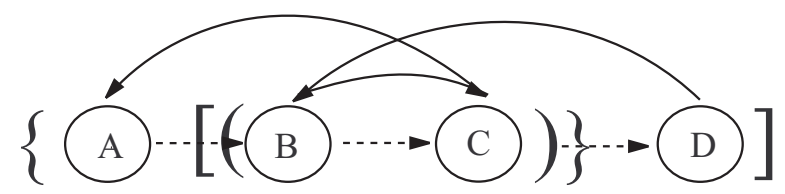

Figure 6: 


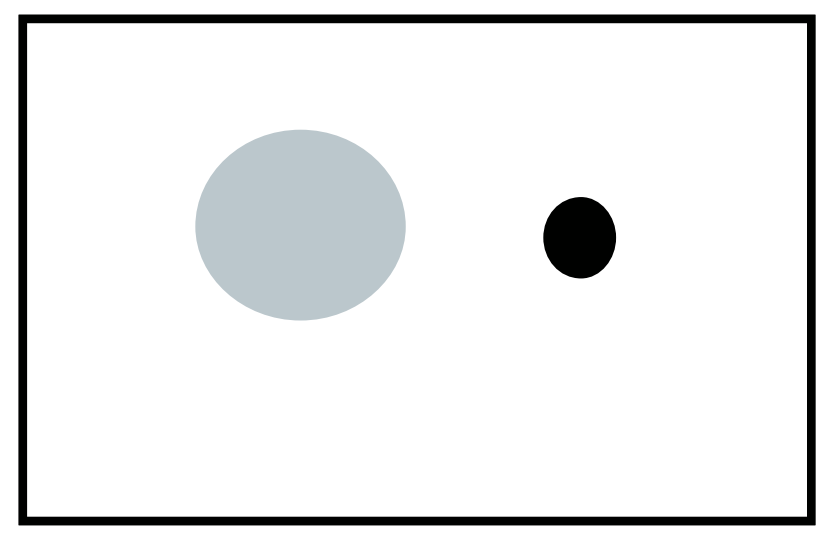

Figure 7: 


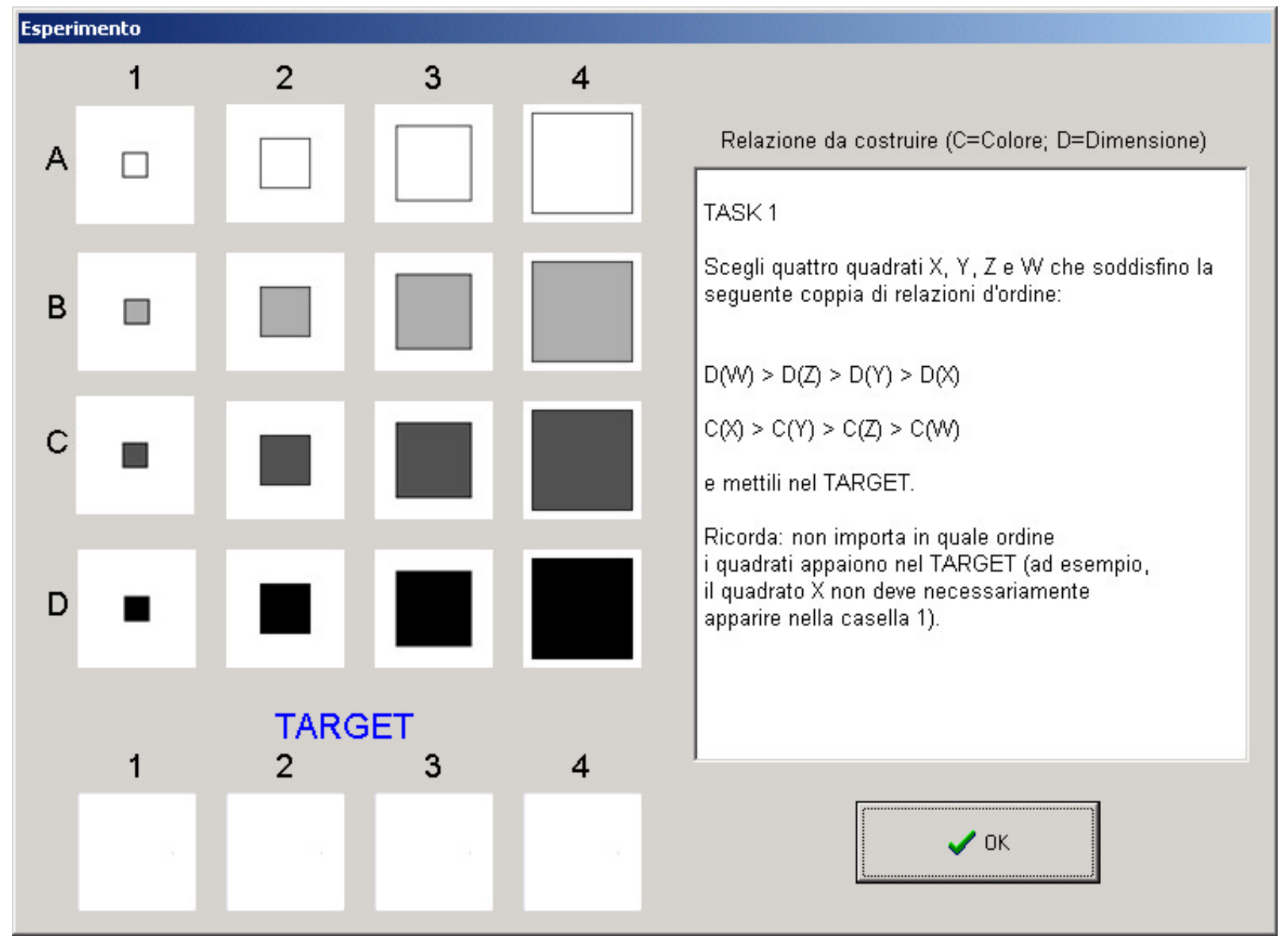

Figure 8: 